\title{
MODELLING THE MECHANICAL RESPONSE OF TWO-LAYERED ARTERY USING THERMOMECHANICAL ANALOGY APPROACH
}

\author{
Janez, Urevc, Vojko Flis, Milan Brumen, Boris Štok
}

Original scientific paper

This work deals with the prediction of the mechanical response of a section of a human common carotid artery (CCA). The arterial residual stress state is accounted for using the thermomechanical analogy (TMA) approach, which is applied in this work to model the mechanical response of a two-layered arterial structure. The starting point to model the arterial residual stress state is normally the cut-open section, which is in the case of patient-specific artery not known. With TMA approach, however, instead of using the arterial zero-stress cut-open configuration to predict the arterial residual stress state, a thermomechanical model of the CCA is considered with its zero-stress geometry defined based on the actual CCA in vivo configuration. The approximation to the CCA residual stress state is then obtained by exposing the auxiliary CCA model to a volumetric deformation, enforced via adequate thermal dilatations. The approach is validated on a circular arterial model and by predicting the CCA cut-open zero-stress state.

Keywords: common carotid artery; finite element method; residual stresses; thermomechanics

Modeliranje mehaničkog odziva dvoslojne stijenke arterije uporabom termo-mehaničke analogije

Izvorni znanstveni članak Ovaj rad obrađuje problem predviđanja mehaničkog odziva dijela zajedničke arterije glave (ZAG). Zaostala naprezanja u arteriji uzeta su u obzir uporabom termo-mehaničke analogije (TMA), koja se primjenjuje u ovom radu za potrebe modeliranja mehaničkog odziva dvoslojne strukture stijenke arterije. Obično se modeliranju zaostalih naprezanja u arteriji pristupi s uzdužno otvorenim modelom arterije, tzv. cut-open section, koji u slučaju bolesnikove arterije nije poznat. S TMA pristupom, umjesto uporabe uzdužno prerezane stijenke arterije, koja doduše osigurava početno stanje bez zaostalih naprezanja, u termo-mehaničkom modelu zajedničke arterije glave također je postignuto početno stanje bez zaostalih naprezanja ali na modelu stvarne, in vivo arterije. Tim pristupom, zaostalo naprezanje u ZAG aproksimirano je podvrgavanjem ZAG modela obujamskom deformacijom, t.j. primjenom odgovarajućih termičkih dilatacija. Takav pristup potvrđen je na modelu arterije kružnog presjeka i postizanjem stanja bez naprezanja u slučaju uzdužnog rezanja stijenke.

Ključne riječi: metoda konačnih elemenata; termo-mehanika; zajednička arterija glave; zaostala naprezanja

\section{Introduction}

Mechanical response of the arterial wall has been shown to participate in pathogenesis of atherosclerosis [1]. An efficient way to study arterial wall mechanics is by means of numerical models. However, due to complex behaviour of the artery and its irregular geometry, several simplifications normally need to be adopted in treating a patient-specific artery.

Common carotid artery (CCA) can be at healthy young individuals treated as a two-layered structure, composed of intima-media and adventitia layer [2]. Both layers exhibit anisotropic material response and are at physiological conditions subject to the so-called prestresses and residual stresses (RS). Pre-stresses represent stresses in the arterial wall due to blood pressure, whereas RSs arise from non-uniform growth and remodelling processes during development of a human individual [3]. For a patient-specific artery RSs cannot be experimentally obtained in non-invasive manner and are thus unknown, however, it is commonly accepted that for realistic prediction of arterial stress state RSs should be accounted for [4].

The effect of RSs on the biomechanical response of arteries is well documented and discussed in literature [5, 6]. If either circumferential or longitudinal strips are excised from a load free arterial tube their curvature will change [4], which indicates multi-dimensional nature of RSs. For the in vivo observed artery the cut-open state represents only a hypothetical (imaginary) state, since the artery cannot be cut out from the body. In order to assess RSs and to determine the arterial material parameters, the uniform strain hypothesis is normally adopted [4, 7]. The hypothesis suggests that cells living at different locations across the vessel wall experience the same mechanical environment [8], which results in a presumption that at physiological conditions the transmural distribution of circumferential strain is uniform.

Residual stress state can be predicted by starting the numerical computation from the arterial cut-open (zerostress) configuration, as presented in $[9,10]$. However, in case of patient-specific artery this approach is practically impossible. In spite of that, the most widely used approach to model patient-specific arterial response, in particular when studying the change of arterial response with respect to various hemodynamic conditions or to stent implementation, is still to take the arterial unloaded configuration as its initial zero-stress state [11, 12], and thus neglect RSs completely. The initial stress-free configuration of the artery is thereby obtained by properly scaling the arterial in vivo configuration. One of somewhat better approaches is using an inverse formulation of the elastostatic equilibrium problem [12], where the stresses in a given deformed state are predicted based on the deformed configuration and the corresponding blood pressure. The approach, however, enables only a prediction of the arterial pre-stresses (and not RSs). Another widely used approach for predicting the arterial pre-stresses is based on the concept introduced by Gee et al. [11], which uses a multiplicative split of the deformation gradient to produce a displacement-free prestressed in vivo configuration.

To incorporate RSs in patient-specific arteries, Alastrué et al. [13] applied a simplified initial strain field, 
obtained from the opening angle problem solution for cylindrical geometries, to actual in vivo (closed) arterial geometry. The applied strain field is however noncompatible with a real non-cylindrical arterial geometry. Polzer et al. [4] recently presented an algorithm which predicts RSs through volumetric tissue growth. The approach is designed to determine such gradient of RS that the homogeneous stress hypothesis is satisfied. The procedure is performed in an iterative manner which can become somewhat time consuming. In addition, the initial arterial configuration, on which RSs are iteratively applied, cannot be determined and has to be obtained in some other way. Schröder and Brinkhues [14] analysed gradients of suitable invariant stress measures in the thickness direction of the arterial wall. RSs were then obtained by smoothing these gradients between the inner and outer margins of individual arterial layer (which was divided further into individual radial sections) using their mean volumetric values. Another approach to determine RSs is by stimulating growth and remodelling processes. Such works can be found in $[3,15,16]$, however, their application is demonstrated only on circular arterial geometries.

Recently, the use of thermomechanical analogy (TMA) approach to model the arterial residual stress state has been presented by the authors [17] where an idealised single-layered model of an artery has been treated. The aim of the present work is to apply the TMA approach to predict the mechanical response of a two-layered human CCA. For that purpose an auxiliary thermomechanical (TM) model of the CCA is constructed with its zero-stress geometry defined based on the actual CCA in vivo geometry. By enforcing volumetric dilatations into this model (meaning, by properly shrinking the model via thermal dilatations) an approximation to the CCA residual stress state is obtained. The key step of the TMA approach, however, is to properly define the properties of the TM model. For this, the mechanical response of a circular arterial segment, corresponding to the observed artery, is needed. This can be obtained based on the data provided from literature or, as in our case, using experimental data along with some in vitro assumptions and solving an identification problem (as, for instance, presented by Stålhand [18]).

In the following sections (sections 2 and 3.1), first, the concept of the TMA approach as well as its purpose is presented in a somewhat simple and easy-to-understand example. Then, in section 3.2 ("Applying TMA approach to an arterial segment") the concept of using the TMA approach to treat an arterial segment is presented. The main part of the paper follows in section 4 ("Case study Treating a patient-specific arterial segment") where a segment of a human CCA is treated with the purpose to predict its in vivo stress state. The predicted CCA RSs and the in vivo stresses are presented in section 4.4 where the approach is also validated. This is performed on a circular arterial segment and by predicting the cut-open state of the CCA.

\section{Setting the problem solution strategy}

In attempting to assess the stress state in a patientspecific artery we are confronted with a series of issues resulting directly from the nature of the problem and therein incorporated uncertainties and missing information. While by nature in the considered boundary value problem (BVP) the mechanical response is normally treated as elastic, the only true information known about the artery is its configuration in the loaded state and the applied pressure load. In order to allow a determination of the respective stress state, the artery's stress-free configuration (or any intermediate configuration with appertaining stress state) and the material behaviour should be given. However, when treating a patient-specific artery, none of them is specified in advance. In fact, it is feasible to solve the problem of stress determination only when ideal cylindrical geometry is accounted for and along with some empirical evidence of the problem.

Based on the above conclusion we set up the following supposition regarding the solution of the stress determination problem in a patient-specific artery: If the problem solution can be found for the case of ideal cylindrical geometry, then a reasonably fair approximation to the stress state in a patient-specific artery can be obtained by a corresponding statically and kinematically consistent mapping of the ideal case solution.

Along with physical reliability of the above statement, the following must be fulfilled: i) existence of the ideal case solution, and ii) existence of appropriate mapping that will allow to obtain the solution of the patient-specific artery problem based on the ideal case solution. In this regard, two major questions vital for setting the problem solution strategy arise: i) How the actual patient-specific arterial geometry can be associated with the ideal cylindrical geometry? and ii) How the ideal case solution should be mapped onto the patient-specific artery without violating static and kinematic consistency of the problem? While the former seems to be manageable in a rather simple way, with the real case geometry substituted by the ideal case geometry on a basis of some equivalence principle, giving proper answer to the latter is a highly demanding task which we will try to resolve applying the TMA approach.

Considering the stated above, the strategy for the solution of the stress determination problem in a patientspecific artery can be divided basically into the following three steps:

Step 1: Establish the ideal case geometry from the given real case geometry.

Step 2: Solve the corresponding mechanical BVP for the ideal case.

Step 3: Assess the original mechanical BVP solution by means of mapping of the corresponding ideal case solution.

Numerous specific activities performed within step 3 , in this text generally referred to as mapping of the ideal case solution, though literally not true or only partially true, are based on the TMA approach. Accordingly, step 3 can be further divided into three main parts:

Sub-step 3.1: Build a thermomechanical analogue (or mapping) model for the ideal case.

Sub-step 3.2: Assess the stress state (in one of the problem's configuration) on the real case geometry using the derived thermomechanical analogue model. 
Sub-step 3.3: Assess the original mechanical BVP solution based on the stress state obtained in sub-step 3.2.

Because understanding of the TMA approach is crucial for the solution of the considered mechanical BVP, the concept of the approach and the steps required therein will be presented first by considering a demonstrative example.

\section{General concepts behind using the TMA approach}

In this section, the TMA approach is presented together with general concepts and reasons for its application by considering a beam bending problem. Although in no direct relation with the problem of an observed arterial section per se, it will help us to highlight all the key steps of the methodology in a somewhat simple and easy-to-understand example. How much similar the two problems actually are from the methodological point of view will be demonstrated in section 3.2, with the TMA approach applied to an idealised arterial segment.

In order to understand well the role of the TMA approach in the solution of the mechanical BVP, all three steps in the BVP solution procedure must be addressed. In this section, however, our focus is primarily on step 1 and step 3 , the steps establishing correspondence between the ideal and real case. Step 2, the step dealing with solving of the ideal case mechanical BVP will be therefore addressed as short as possible, underlining however characteristic features of the solution procedure.

\subsection{Stress assessment in a beam bending problem 3.1.1 Problem description}

Let us observe an initially curved beam of a rectangular cross-sectional area in its straightened and stretched configuration $\Omega_{2}$ (Fig. 1a). The goal that we want to achieve in this section is to determine the stresses in observed beam's configuration $\Omega_{2}$. In this configuration, which is the only known configuration of the problem, the beam is characterized by its length $L_{2}$, variable cross-sectional area $A_{2}(x)$ and height $h_{2}(x)$. The magnitude of the pulling force is assumed to be known, its value being $F_{0}$. For the sake of simplicity let us assume incompressible and linearly elastic material behaviour with the known value of Young's modulus $E_{0}$. Let us also assume that no further information is known except some empirical evidence relating to the distribution of stresses, specific to the nature of the considered problem.

The nature of the mechanical response of the beam is the following: by removing $F_{0}$, the beam shrinks in the axial $(x)$ direction and expands in the transversal $(z)$ direction. The obtained configuration $\Omega_{1 \lambda}$, which is characterized by the length $L_{1}$, cross-sectional area $A_{1}(x)$ and height $h_{1}(x)$, is not yet stress free, because the zerostress configuration $\Omega_{0}$ is actually represented by the curved beam in the absence of any externally applied load (see Fig. 1a). While it is obvious that the stresses in the configuration $\Omega_{1 \lambda}$ (referred to also as residual stresses in the case of artery problem) are caused by bending of the curved bar, their magnitude, however, is not known, because the initial configuration $\Omega_{0}$ of the beam is not known (i.e., given). In the stress-free configuration $\Omega_{0}$ the beam is characterized by the length $L_{0}$, cross-sectional area $A_{0}(x)$, height $h_{0}(x)$ and radius of curvature $R_{0}(x)$.

Because the initial stress-free configuration of the beam is not known, the stresses in any other configuration of the beam cannot be computed. In the following, to obtain an approximate solution of the problem, we proceed in accordance with the problem solution strategy and respective steps in the solution procedure, described in the previous section. $\mathbf{a}$

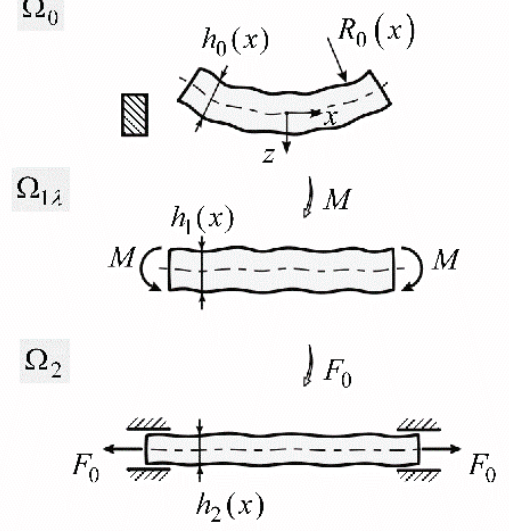

BEAM OF REAL GEOMETRY

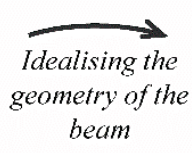
beam

Figure 1 Configurations of the beam example: (a) the observed "real case" problem, and (b) the "ideal case" substitution problem

\subsubsection{Idealising the geometry of the beam, Step 1}

In order to obtain an estimation to the mechanical response of the investigated beam, the observed real case problem (Fig. 1a) is substituted by an ideal case problem, obtained by idealising beam's geometry (Fig. 1b). The idealisation consists of assuming that the longitudinal axis of the beam in its loaded state $\Omega_{2}^{\mathrm{C}}$ is a straight line, and that the cross-sectional area and its height are constant, thus: $A_{2}^{\mathrm{C}}(x)=\tilde{A}_{2}$ and $h_{2}^{\mathrm{C}}(x)=\tilde{h}_{2}$. The idealisation further implies that the beam's geometry in the stress-free 
configuration $\Omega_{0}^{\mathrm{C}}$ is characterised by a single radius of curvature with as-yet-unknown radius $\tilde{R}_{0}$.

\subsubsection{About solving the ideal case BVP, Step 2}

In the absence of knowing the radius $\tilde{R}_{0}$ additional information characterising the problem is needed to solve the BVP. In this regard, let us suppose that, by some phenomenological reason evidenced empirically, the gradient of the stress field is known for the final configuration of the beam. The discussed specifics of the stress distribution may be referred as the hypothesis of known stress gradient distribution ${ }^{1}$. In Fig. 1b, the slope of the stress distribution in the loaded configuration $\Omega_{2}^{\mathrm{C}}$ is specified by the angle $\varphi_{0}$. Acquiring knowledge about the angle $\varphi_{0}$ makes the ideal case problem solvable. The radius $\tilde{R}_{0}$ can be easily determined by using the above hypothesis, whereas the remaining parameters $\tilde{L}_{0}, \tilde{A}_{0}$ and $\tilde{h}_{0}$ are obtained from the incompressibility condition by taking the complete unloading of the beam into account. Based on these results, the mechanical response of any intermediate configuration between $\Omega_{0}^{\mathrm{C}}$ and $\Omega_{2}^{\mathrm{C}}$ in Fig. 1b can be determined. Thus, in the configuration $\Omega_{1 \lambda}$ the stress distribution is $\sigma_{x}(x, z)=-\tan \left(\varphi_{0}\right) z$.

\subsubsection{Introducing thermomechanical analogue model, Sub-step 3.1}

In the following, let us look for an alternative approach to solve the ideal case BVP. This may at first seem redundant, but it is essential in order to solve the original real case BVP. Namely, the problem that arises is, how the obtained mechanical response of the idealised beam (i.e., either from states $\Omega_{2}^{\mathrm{C}}$ or $\Omega_{1 \lambda}^{\mathrm{C}}$ ) can adequately be mapped onto the beam of real geometry (Fig. 1a). Unfortunately, it can be easily demonstrated that direct scaling based on the change in geometry is not adequate, because it leads either to stress non-equilibrium or strain incompatibility. In this work, therefore, the the TMA approach is used.

The first step in the TMA approach is setting up of a thermomechanical analogue model capable of reproducing the same stress distribution and configuration as established by the $\Omega_{1 \lambda}^{\mathrm{C}}$ state, without the bending (Fig. 2 ). From the field of continuum thermomechanics it is known that the state $\Omega_{1 \lambda}^{\mathrm{C}}$ (i.e., its configuration and stresses; Fig. 2a) can also be established by enforcing appropriate thermal (volumetric) dilatation in a clampedclamped straight beam, with no end rotation allowed, but free to extend in the longitudinal direction, as presented in Fig. 2b. More specifically, by exposing the beam in its

\footnotetext{
${ }^{1}$ As mentioned, it is presumed that this is a well-known hypothesis of the observed beam problem, similarly as the uniform strain hypothesis in arteries, where $\varphi_{0}=0$ for circumferential strains at the mean blood pressure.
}

stress-free configuration $\Psi_{0}^{\mathrm{C}}$ to the temperature field resulting from the imposed temperature difference $\Delta T_{\mathrm{a}}$ and $\Delta T_{\mathrm{b}}$, applied respectively on the upper and lower surface of the beam, the targeted stress distribution and configuration can be obtained in the thermally deformed configuration $\Psi_{1 \lambda}^{\mathrm{C}}$. Namely, a linear temperature distribution over the cross-section, which is obtained as the solution of the specified steady-state heat transfer $\mathrm{BVP}$, gives rise, under the given mechanical boundary conditions and assuming linear elastic behaviour, to a linear stress distribution over the cross-section. The introduced analogue model will be referred henceforth as the thermomechanical analogue model, shortly the TM model.

From the point of ensuring consistent mapping we are looking for, the conceived TM model is advantageous because it is essentially a fictitious model, meaning that we can chose its initial geometry arbitrarily, in principle. Thereby the material properties of the model as well as the thermal loading need to be determined accordingly, i.e., in such a way to establish correspondence with the initial configuration of the TM model $\Psi_{0}^{\mathrm{C}}$ and the targeting state $\Omega_{1 \lambda}^{\mathrm{C}}$.

The most reasonable decision in defining the initial configuration of the TM model is to adopt the geometry of the beam in its loaded state $\Omega_{2}^{\mathrm{C}}$. Namely, this is the only geometry in the considered beam problem that is given from the very beginning (which is also the case when treating patient-specific arteries, as seen later).

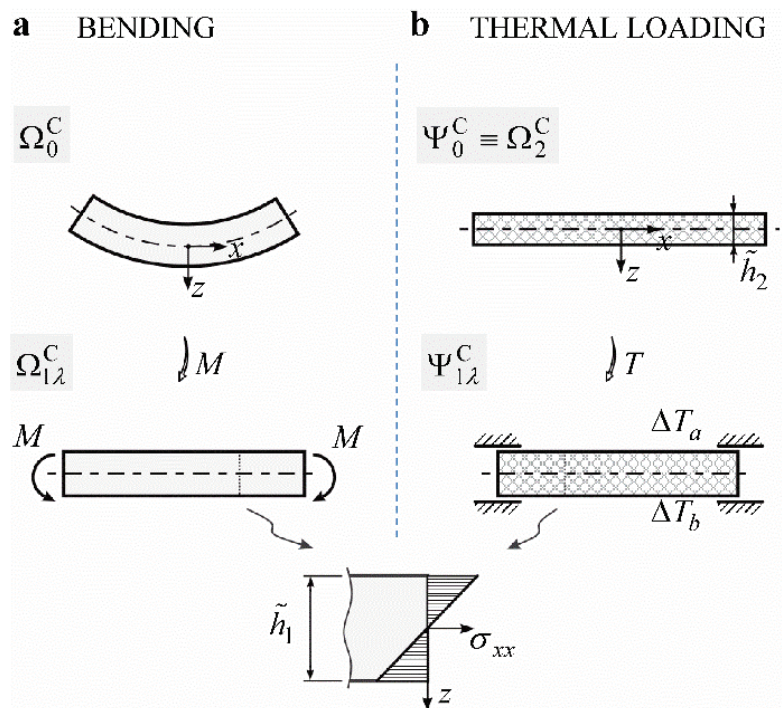

Figure 2 Stress distribution in the beam configuration $\Omega_{1 \lambda}^{\mathrm{C}}$, obtained: a by bending initially curved beam from its stress-free configuration $\Omega_{0}^{\mathrm{C}}$, and $\mathbf{b}$ by enforcing thermal dilatation in stress-free configuration $\Psi_{0}^{\mathrm{C}}$ of the TM model

Taking into consideration that the TM model is actually an auxiliary model, its material properties are not required to be in any relation with material of the observed beam problem per se. So, the purpose now is to determine the properties of the TM model in such a way, 
that the configuration and the stresses in its loaded state $\Psi_{1 \lambda}^{\mathrm{C}}$ will match the state $\Omega_{1 \lambda}^{\mathrm{C}}$ of the idealised beam.

Given to the nature of boundary conditions prescribed on the TM beam (no end rotation and free longitudinal extension, Fig. 2b), the beam does not deflect when exposed to linear change in the temperature $\Delta T(x, z)=\Delta T_{\mathrm{m}}+\Delta \vartheta_{z}$ and a linear distribution of stresses originate. Here, $\Delta T_{\mathrm{m}}=\left(\Delta T_{\mathrm{b}}+\Delta T_{\mathrm{a}}\right) / 2$ is the mean temperature change and $\Delta \vartheta=\left(\Delta T_{\mathrm{b}}-\Delta T_{\mathrm{a}}\right) / \tilde{h}_{2}$ is the temperature gradient.

Determination of the TM model material properties is subject to the constraints that originate from the above stated matching demands. In particular, the elongation $\Delta L^{\psi}$ and transversal contraction $\Delta h^{\psi}$ of the TM model are prescribed, as well as the stress distribution $\sigma_{x}^{\psi}(x, z)=-\tan \left(\varphi_{0}\right) z$. Considering the respective strain distributions, $\varepsilon_{x}^{\psi}(x, z)$ and $\varepsilon_{z}^{\psi}(x, z)$, and linear elastic behaviour the following constraints must be fulfilled:

$$
\begin{aligned}
& \Delta L^{\psi}(z)=\left[\sigma_{x}^{\psi}(x, z) / E^{\psi}+\right. \\
& \left.\quad+\alpha_{x}^{\psi}\left(\Delta T_{\mathrm{m}}+\Delta \vartheta z\right)\right] L_{0}^{\psi}=\tilde{L}_{1}-\tilde{L}_{2} \\
& \Delta h^{\psi}=\alpha_{z}^{\psi} \Delta T_{\mathrm{m}} h_{0}^{\psi}=\tilde{h}_{1}-\tilde{h}_{2} \\
& \sigma_{x}^{\psi}(x, z)=-\tan \left(\varphi_{0}\right) z
\end{aligned}
$$

where $E^{\psi}$ is Young's modulus and $\alpha_{x}^{\psi}$ and $\alpha_{z}^{\psi}$ are respectively the coefficients of thermal expansion in the axial and transverse direction of the beam (Fig. 3a).

\section{a THERMOMECH. MODEL OF IDEALISED BEAM}

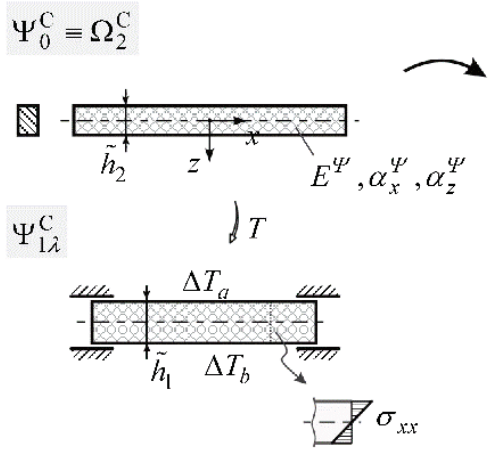

$\Psi_{0} \equiv \Omega_{2}$
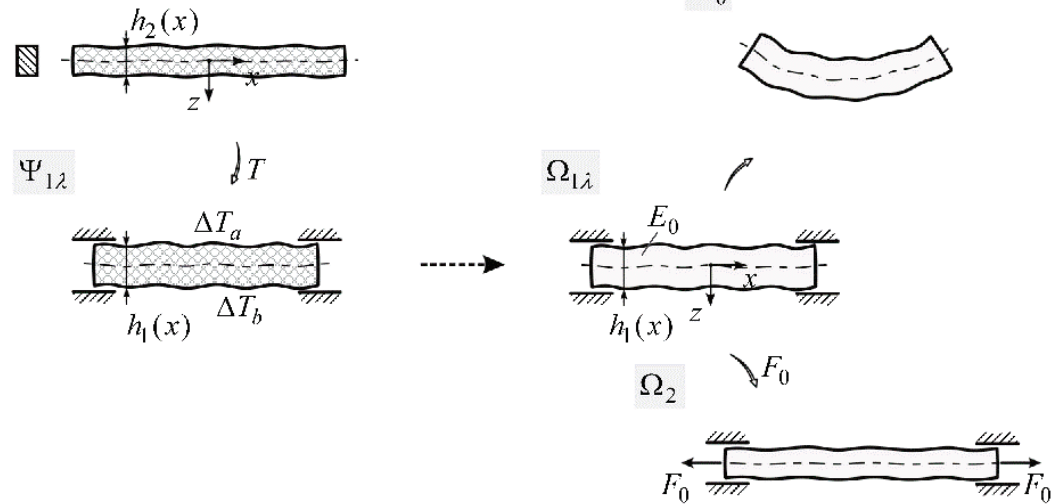

Figure 3 The TMA approach: (a) thermomechanical model of the idealised beam, (b) thermomechanical model of the real beam, and $\mathbf{c}$ mechanical response of the real beam (Fig. 1a)

In principle, as the above system of equations is expressed in terms of five parameters, three material $\left(E^{\psi}, \alpha_{x}^{\psi}, \alpha_{z}^{\psi}\right)$ and two loading $\left(\Delta T_{\mathrm{m}}, \Delta \vartheta\right.$, or equivalently $\Delta T_{\mathrm{a}}, \Delta T_{\mathrm{b}}$ ) parameters, its fulfilment can be achieved under different combination of the respective parameters' values. Having only three equations and five unknowns, a set of selected three parameters can be expressed in terms of the remaining two, with their values chosen arbitrarily. Certain parameter pairs, however, cannot be chosen arbitrarily, e.g. $\left(\alpha_{x}^{\psi}, \Delta T_{\mathrm{m}}\right)$ or ( $\alpha_{x}^{\psi}, \alpha_{z}^{\psi}$ ). From the system of equations (1) it also follows that a meaningful solution is obtained only when $\Delta T_{\mathrm{m}} \neq 0$ and $\Delta \vartheta \neq 0$.

With a consistent combination of the TM model parameters determined, the TM model is completely defined. Because appropriate specification of the TM model is vital for solving the original real case BVP, let us remind what principal characteristics of the conceived model are.
Firstly, the TM model stress-free configuration $\Psi_{0}^{\mathrm{C}}$ (Fig. 2a) is identical to the ideal case configuration $\Omega_{2}^{\mathrm{C}}$ which represents the beam in its loaded state (Fig. 1b). Secondly, the boundary conditions in the TM model are prescribed in a way that conditions on the boundary, characterising the ideal case intermediate configuration $\Omega_{1 \lambda}^{\mathrm{C}}$ state, are attained by thermal loading. Thirdly, the TM model material and thermal loading parameters are specified so that in its loaded configuration $\Psi_{1 \lambda}^{\mathrm{C}}$ the same stress distribution and configuration, as established by the $\Omega_{1 \lambda}^{\mathrm{C}}$ state, is obtained.

\subsubsection{Assessing the real case beam problem by means of the TM model, Sub-step 3.2}

So far we have shown that the idealised beam configuration $\Omega_{1 \lambda}^{\mathrm{C}}$ and corresponding stresses have been calculated by two equivalent approaches, with the application of mechanical loading (bending) and with the 
application of thermal loading using the TMA approach. The ideal case mechanical BVP solution is thereby used to characterise the corresponding TM model.

Now, taking into consideration that by applying the TMA approach a consistent state of stresses and strains is achieved by definition, it could be in the same manner applied to solve the original mechanical BVP. The application of the TMA approach on the real beam geometry, taking the above determined values of the TM model material and loading parameters as well as respective boundary conditions into account, is quite justified when considering phenomenological and geometrical similarity between the two cases (note that the ideal case has been obtained by idealising the geometry of the real case).

First, following the same procedure as above, a new TM model $\Psi_{0}$ (Fig. 3b), with the material properties and associated boundary conditions of the ideal case TM model adopted, is constructed considering the real beam configuration $\Omega_{2}$ (Fig. 1a). The $\Psi_{0}$ configuration is stress-free. Application of the previously determined thermal loads $\Delta T_{\mathrm{a}}$ and $\Delta T_{\mathrm{b}}$ gives rise to a temperature field that obeys the heat transfer equation in the considered real case domain. Finally, in consequence of the established temperature field and given boundary conditions the stresses appear in the TM model, which deforms to the configuration $\Psi_{1 \lambda}$. In this way, i.e. by considering the thermomechanical analogy, a fair approximation of the intermediate $\Omega_{1 \lambda}$ configuration and the corresponding stress state for the real case beam problem is obtained.

The strains obtained by the TM model, however, cannot be considered as an approximation to the strains of the $\Omega_{1 \lambda}$ configuration, because neither the configuration $\Psi_{0}$ resembles the zero-stress configuration $\Omega_{0}$ nor the strains are related to stresses by the true constitutive law.

\subsubsection{Assessing the real case beam problem by means of the TM model, Sub-step 3.3}

Having determined the $\Omega_{1 \lambda}$ configuration and corresponding stress distribution we are now able to assess correctly the state of strains in the $\Omega_{1 \lambda}$ configuration. This is performed by considering the actual material behaviour of the beam. Practically, this is obtained by switching the material properties and loadings of the TM model in the configuration $\Psi_{1 \lambda}$ ( $\Psi_{1 \lambda}=\Omega_{1 \lambda}$ ) to real material properties and loadings (Fig. 3c). With such a model obtained, releasing the stresses completely defines the zero-stress configuration $\Omega_{0}$, whereas applying the pulling force $F_{0}$ yields the stresses in the given loaded configuration $\Omega_{2}$, the specified problem task being thus finally solved.

\subsection{Applying TMA approach to an arterial segment 3.2.1 Problem description and its solution}

The subject of observation is a real arterial segment in its loaded (in vivo) state $\Omega_{2}$. For the sake of simplicity, let us assume that the longitudinal axis of the segment is straight and that the shape of the segment's cross section is constant (Fig. 4a). Additionally, because of time variation of blood pressure $P^{\mathrm{CA}}(t)$, which is assumed to be known, it follows that $\Omega_{2}=\Omega_{2}(t)$. The artery is considered as a two-layered structure, composed of intima-media (IM) and adventitia $(A)$ layer. The adopted constitutive behaviour is hyperelastic, isotropic and incompressible. Further, there exists some empirical evidence regarding the stress and strain distribution in the loaded state, specific to the nature of the considered problem, which is also assumed known. The purpose in the following is to determine the stress state in the loaded configuration $\Omega_{2}$ of the observed arterial segment.

In order to find the solution of the problem, the corresponding BVP must be formulated first. From empirical evidence, the mechanical response of a real arterial segment is the following (Fig. 4a). In the loaded configuration $\Omega_{2}$, the artery is loaded with blood pressure $P^{\mathrm{CA}}$. By removing the pressure, the artery shrinks in the radial direction and takes the so called stretched configuration $\Omega_{1 \lambda}$. By further cutting the artery transversely, the artery shrinks in the axial direction (for the stretch $\lambda$ ) and takes the unloaded configuration $\Omega_{1}$. In this state, although the artery is free of external loads, it is still subject to internal stresses. Finally, by cutting the artery longitudinally, it springs-open and takes the zerostress configuration $\Omega_{0}$. In reality, due to different natural causes originated from the artery's growth, the stresses in the two-layered structure need not to vanish completely. For the purpose of this work we will assume, however, that in the zero-stress configuration $\Omega_{0}$ the two layers are stress free, meaning that the same opening angle $\Theta_{0}^{I M}=\Theta_{0}^{A}=\Theta_{0}$ would characterize both layers if they were delaminated.

It is clear that even in the case the material behaviour of both layers were specified, a determination of the respective stress state in the loaded configuration $\Omega_{2}$ is not possible, because the artery's stress-free configuration $\Omega_{0} \quad$ (or any intermediate configuration with the appertaining stress state) is not known. To solve the problem nevertheless, the empirical evidence specific to the stress and strain distribution of the arterial loaded state should be accounted for. How this additional information is considered in the BVP solution procedure will be presented in section 4 ("Case study - Treating a patientspecific arterial segment"), where the case of a patientspecific artery will be computationally analysed.

For the same reasons as discussed in the demonstrative beam example case, we consider the TMA 
approach adequate to find an approximate solution to the considered real arterial segment problem. We therefore proceed in accordance with the problem solution strategy and respective steps, described in section 2 ("Setting the problem solution strategy").

Because step 1 (simplifying/idealising the geometry of the real artery, Fig. 4b) and sub-steps 3.2 and 3.3 (mapping the stresses of the idealised artery to the real artery) of the solution procedure are performed computationally in the same manner as in the beam example, the emphasis will be given to a detailed description of the topics related to step 2 and sub-step 3.1. The specifics regarding the solution procedure steps that are by-passed here will be thoroughly addressed in section treating the patient-specific arterial segment (section 4).

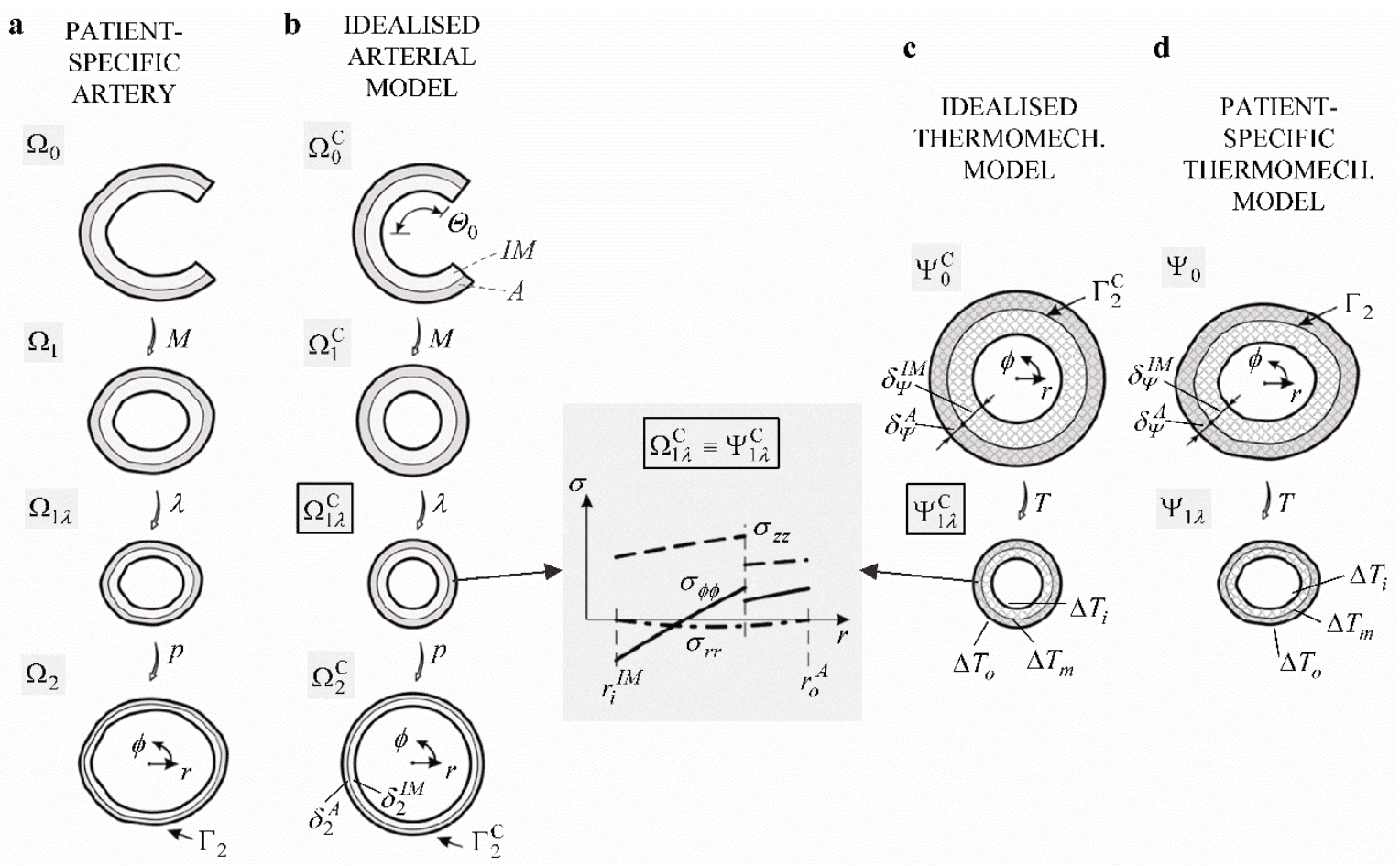

Figure 4 Predicting the mechanical response of a patient-specific arterial segment $\Omega_{2}$ : (a) patient-specific arterial segment, (b) idealised arterial segment, (c) idealised thermomechanical arterial segment, and (d) thermomechanical model of patient-specific arterial segment

\subsubsection{Treating the idealised arterial segment, Step 2}

To estimate the mechanical response of the arterial segment, its cross-sectional shape is idealised into a circular one. The geometry of the circular arterial segment in the loaded configuration $\Omega_{2}^{\mathrm{C}}$ is thus defined by the outer radius of the adventitia layer $r_{o}^{A}$ and the layer thicknesses $\delta_{2}^{A}$ and $\delta_{2}^{I M}\left(\delta_{2}^{A}+\delta_{2}^{I M}=\delta_{2}\right)$. In the cutopen zero-stress configuration $\Omega_{0}^{\mathrm{C}}$ the segment is defined with the outer radius of the adventitia layer $R_{o}^{A}$, the layer thicknesses $\delta_{0}^{A}$ and $\delta_{0}^{I M}\left(\delta_{0}^{A}+\delta_{0}^{I M}=\delta_{0}\right)$, and the opening angle $\Theta_{0}$. The lengths of the arterial segment in those configurations are respectively $L_{2}$ and $L_{0}$.

In setting the BVP equations for the circular arterial segment the above described characterisation of the arterial mechanical response is taken into account. The deformation and respective principal stretches of a material particle from the zero-stress configuration $\Omega_{0}^{C}$ to the loaded configuration $\Omega_{2}^{\mathrm{C}}$ are characterised as follows. Closing of the cut-open artery (configuration $\Omega_{1}^{\mathrm{C}}$ ) is defined by the opening angle $\Theta_{0}$ and the axial stretch $\Lambda$, which is followed by additional axial stretch $\lambda$, when reinserting the arterial segment in the human's body (configuration $\Omega_{1 \lambda}^{\mathrm{C}}$ ). Finally, the in vivo state (configuration $\Omega_{2}^{\mathrm{C}}$ ) is obtained by applying the blood pressure $P^{\mathrm{CA}}$ on the inner surface of the $I M$ layer. Although the blood pressure varies with time $P^{\mathrm{CA}}(t)$, the problem can be treated as quasi-static [19]. Therefore, we will denote the time dependence in the following equations only occasionally.

The material particles located on the circumference $r=r(t)$ in the configuration $\Omega_{2}^{\mathrm{C}}(t)$ are supposed to take the circumferential position defined by the reference radius $R(r)$ in the configuration $\Omega_{0}^{\mathrm{C}}$. From the incompressibility condition the following correspondence relation is obtained:

$R(r)=\sqrt{\left(R_{o}^{A}\right)^{2}-\frac{\pi \lambda \Lambda}{\Theta_{0}}\left(\left(r_{o}^{A}\right)^{2}-r^{2}\right)}$

where: $r_{o}^{A}-\delta_{2} \leq r \leq r_{o}^{A}$.

When all three successive motions of the particle (from $\Omega_{0}^{\mathrm{C}}$ to deformed configurations $\Omega_{1}^{\mathrm{C}}, \Omega_{1 \lambda}^{\mathrm{C}}$ and 
$\Omega_{2}^{\mathrm{C}}$; Fig. 4b) are accounted for, the deformation of the material particles in the loaded configuration $\Omega_{2}^{\mathrm{C}}$ is defined by the principal stretches:

$\lambda_{r}(r)=\frac{\Theta_{0} R(r)}{\pi r \lambda \Lambda}, \quad \lambda_{\phi}(r)=\frac{\pi r}{\Theta_{0} R(r)}, \quad \lambda_{z}(r)=\lambda \Lambda$.

Above, $\lambda_{r}, \lambda_{\phi}$, and $\lambda_{z}$ are respectively the radial, circumferential and axial principal stretch.

The stress state in the two arterial layers, $s \in\{I M, A\}$, resulting from the described deformation (3) is expressed by the Cauchy stress tensor $\boldsymbol{\sigma}$, which for an isotropic incompressible material reads:

$$
\boldsymbol{\sigma}^{s}=-p^{s} \mathbf{I}+\overline{\boldsymbol{\sigma}}^{s}=-p^{s} \mathbf{I}+2 \mathbf{F}^{s} \frac{\partial W^{s}}{\partial \mathbf{C}^{s}} \mathbf{F}^{s \mathrm{~T}} ; s \in\{I M, A\}
$$

In the equation $p^{\mathrm{s}}$ is the Lagrange multiplier that enforces incompressibility, $\mathbf{I}$ is the identity tensor and $\mathbf{F}^{\mathbf{S}}$ is the deformation gradient tensor:

$$
\mathbf{F}^{s}=\operatorname{diag}\left[\lambda_{r}(r), \lambda_{\phi}(r), \lambda_{z}(r)\right]
$$

Further, $\mathbf{C}^{s}=\mathbf{F}^{s \mathrm{~T}} \mathbf{F}^{s}$ is the right Cauchy-Green tensor and $W^{S}$ is the strain energy density function. The same functional structure of the strain energy density function is considered for both layers [9]:

$$
W^{s}=\frac{c_{a}^{s}}{c_{b}^{s}}\left[\exp \left(\frac{c_{b}^{s}}{2}\left(I_{1}^{s}-3\right)\right)-1\right]
$$

with $c_{a}^{s}$ and $c_{b}^{s}$ being the constitutive model parameters of the respective layer and $I_{1}^{S}$ is the first invariant of the tensor $\mathbf{C}^{S}$

When the assumed material behaviour (6) is taken into account, the non-zero components of the stress tensor (4) are expressed as:

$$
\begin{aligned}
& \sigma_{k k}^{s}(r)=-p^{s}(r)+\bar{\sigma}_{k k}^{s}(r) \\
& \bar{\sigma}_{k k}^{s}(r)=\lambda_{k}^{2}(r) c_{a}^{s} \exp \left[\frac { c _ { b } ^ { s } } { 2 } \left(\lambda_{r}^{2}(r)+\lambda_{\phi}^{2}(r)+\right.\right. \\
& \left.\left.+\lambda_{z}^{2}(r)-3\right)\right] ; k=r, \phi, z .
\end{aligned}
$$

Apart from fulfilling the equilibrium equations:

$$
\frac{\mathrm{d} \sigma_{r r}^{s}}{\mathrm{~d} r}+\frac{\sigma_{r r}^{s}-\sigma_{\phi \phi}^{s}}{r}=0
$$

in each layer domain, the respective radial stress distributions have to satisfy the associated boundary conditions: $\sigma_{r r}^{I M}\left(r_{i}^{I M}\right)=-P^{\mathrm{CA}}, \quad \sigma_{r r}^{A}\left(r_{o}^{A}\right)=0$,

and the continuity condition at the layers' interface $r_{o}^{I M}=r_{i}^{A}$ :

$\sigma_{r r}^{I M}\left(r_{o}^{I M}\right)=\sigma_{r r}^{A}\left(r_{i}^{A}\right)$

The symbols " $o$ " and " $i$ " are used respectively to denote the outer and the inner radius of the layer.

The set of equations (2)-(10) completely defines the BVP of the circular arterial segment. Assuming the material behaviour and zero-stress configuration $\Omega_{0}^{\mathrm{C}}$ as known ( $\left.R_{o}^{A}, \delta_{0}^{A}, \delta_{0}^{I M}, L_{0}, \Theta_{0}\right)$, the mechanical response of all of segment's configurations in Fig. $4 \mathbf{b}$ can be determined. The solution of the considered BVP comprises a determination of the loaded configuration $\Omega_{2}^{\mathrm{C}}\left(r_{o}^{A}, \delta_{2}^{A}, \delta_{2}^{I M}, L_{2}\right)$ and the corresponding stress distribution.

In order to enable the stress computation (7), the functional dependence of the Lagrange multipliers $p^{I M}(r)$ and $p^{A}(r)$ has to be determined. From the equilibrium equations (8) the following dependence is obtained after taking the boundary conditions (9) into account:

$$
\begin{aligned}
& p^{I M}(r)=P^{\mathrm{CA}}+\bar{\sigma}_{r r}^{I M}(r)- \\
& -\int_{r_{i}^{I M}}^{r}\left(\bar{\sigma}_{\phi \phi}^{I M}-\bar{\sigma}_{r r}^{I M}\right) \frac{\mathrm{d} r}{r} ; \quad r_{i}^{I M} \leq r \leq r_{o}^{I M} \\
& p^{A}(r)=\bar{\sigma}_{r r}^{A}(r)+\int_{r}^{r_{o}^{A}}\left(\bar{\sigma}_{\phi \phi}^{A}-\bar{\sigma}_{r r}^{A}\right) \frac{\mathrm{d} r}{r} ; \quad r_{i}^{A} \leq r \leq r_{o}^{A}
\end{aligned}
$$

From the stress continuity condition (10) it comes out that the radii $r_{i}^{I M}, r_{o}^{I M}=r_{i}^{A}$ and $r_{o}^{A}$ of the loaded configuration $\Omega_{2}^{\mathrm{C}}$ are interconnected by the relation:

$$
P^{\mathrm{CA}}-\int_{r_{i}^{A}}^{r_{o}^{A}}\left(\bar{\sigma}_{\phi \phi}^{A}-\bar{\sigma}_{r r}^{A}\right) \frac{\mathrm{d} r}{r}=\int_{r_{i}^{I M}}^{r_{o}^{I M}}\left(\bar{\sigma}_{\phi \phi}^{I M}-\bar{\sigma}_{r r}^{I M}\right) \frac{\mathrm{d} r}{r}
$$

from where direct dependence of the loaded configuration $\Omega_{2}^{\mathrm{C}}(t)$ on the applied blood pressure $P^{\mathrm{CA}}(t)$ is manifested. Also, considering that the three radii are constrained by the incompressibility condition, it is evident that in the BVP definition either the blood pressure $P^{\mathrm{CA}}(t)$ or the corresponding configuration $\Omega_{2}^{\mathrm{C}}(t)$, represented e.g. by the radius $r_{o}^{A}(t)$, should be specified.

With the Lagrange multipliers $p^{I M}(r, t)$ and $p^{A}(r, t)$ determined (11), stresses can be computed from 
(7) for any value of the pressure $P^{\mathrm{CA}}(t)$. This obviously enables, when setting in the Eqs. (11) and (12) the pressure to zero, a determination of the stretched configuration $\Omega_{1 \lambda}^{\mathrm{C}}$ and computation of the corresponding (residual) stresses.

\subsubsection{Thermomechanical analogue model of the idealised arterial segment, Sub-step 3.1}

In the TMA approach, setting up of a thermomechanical analogue model capable of reproducing the same stress distribution and configuration, as established in the $\Omega_{1 \lambda}^{\mathrm{C}}$ state of the idealised arterial segment, is the first step. The distribution of RSs in the configuration $\Omega_{1 \lambda}^{\mathrm{C}}$ is characterised by the closing and axial stretching of initially cut-open artery. The circumferential stress $\sigma_{\phi \phi}$ is affected primarily by bending, whereas the axial stress $\sigma_{z z}$ is affected by stretching (see for instance [20]). Similarly as in the beam bending example, such a mechanical response can be obtained when a two-layered cylindrical (TM) tube segment, clamped at both ends, is exposed to adequate temperature change, Fig. 4c. More specifically, by exposing the tube segment in its stressfree configuration $\Psi_{0}^{\mathrm{C}}$ to a temperature field resulting from the imposed temperature difference $\Delta T_{i}, \Delta T_{o}$ and $\Delta T_{m}$ (Fig. 4c), the targeted stress distribution and configuration can be obtained in the thermally deformed configuration $\Psi_{1 \lambda}^{\mathrm{C}}$. The two layers of the TM model are supposed to be defined in a way to give the mechanical response similar to the one, experienced by the $I M$ and $A$ layer of the circular arterial segment.

In view of later application of the introduced TM model to the analysis of the real artery, the elimination of the closing up of the (initially) cut-open section is advantageous. In the case of idealised arterial segment, all deformed configurations of the TM model are actually cylindrical. The logical step is now to adopt the in vivo configuration $\Omega_{2}^{\mathrm{C}}$ as the TM model's stress-free configuration $\Psi_{0}^{\mathrm{C}}$. Since the initial and final configuration of the TM model are given, its material properties and thermal loading have to be properly defined in order to obtain stress distribution and configuration as obtained in the targeting $\Omega_{1 \lambda}^{\mathrm{C}}$ state.

In order to catch the targeted $\sigma_{\phi \phi}$ and $\sigma_{z z}$ distributions by the TM model, the assumed material behaviour has to be cylindrically transversely isotropic. Additionally, since the RSs are small in comparison to the stresses in the arterial loaded (in vivo) state, the material behaviour of the TM model is assumed linearly elastic. Considering the TM model is not allowed to deform in the axial direction, the stress state caused by the change of the temperature field $T(r)$ can be expressed in terms of the radial displacement $u(r)$ [17], as follows:

$$
\begin{aligned}
& \sigma_{r r}^{s}=A_{\mathrm{P}}^{s} \frac{\mathrm{d} u^{s}}{\mathrm{~d} r}+A_{\mathrm{PT}}^{s} \frac{u^{s}}{r}-\beta_{\mathrm{P}}^{s} T^{s} \\
& \sigma_{\phi \phi}^{s}=A_{\mathrm{PT}}^{s} \frac{\mathrm{d} u^{s}}{\mathrm{~d} r}+A_{\mathrm{P}} \frac{u^{s}}{r}-\beta_{\mathrm{P}}^{s} T^{s} ; s \in\left\{I M^{\Psi}, A^{\Psi}\right\} \\
& \sigma_{z z}^{s}=A_{\mathrm{TP}}^{s}\left(\frac{\mathrm{d} u^{s}}{\mathrm{~d} r}+\frac{u^{s}}{r}\right)-\beta_{\mathrm{P}}^{s} T^{s}
\end{aligned}
$$

with the following relationships between the parameters that characterise the respective material behaviour with respect to "in-plane" (P) and transverse (T) direction (elastic moduli $E_{\mathrm{P}}^{s}, E_{\mathrm{T}}^{s}$, Poisson ratios $v_{\mathrm{P}}^{s}, v_{\mathrm{TP}}^{s}$ and $v_{\mathrm{PT}}^{S}$, coefficients of thermal expansion $\left.\alpha_{\mathrm{P}}^{S}, \alpha_{\mathrm{T}}^{s}\right)$ defined as:

$$
\begin{aligned}
& A_{\mathrm{P}}^{s}=\frac{E_{\mathrm{P}}^{s}\left(1-v_{\mathrm{TP}}^{s} v_{\mathrm{PT}}^{s}\right)}{D^{s}}, A_{\mathrm{PT}}^{s}=\frac{E_{\mathrm{P}}^{s}\left(v_{\mathrm{P}}^{s}+v_{\mathrm{TP}}^{s} v_{\mathrm{PT}}^{s}\right)}{D^{s}}, \\
& A_{\mathrm{TP}}^{s}=\frac{E_{\mathrm{P}}^{s}\left(v_{\mathrm{TP}}^{s}+v_{\mathrm{P}}^{s} v_{\mathrm{TP}}^{s}\right)}{D^{s}}, A_{\mathrm{T}}^{s}=\frac{E_{\mathrm{T}}^{s}\left(1-\left(v_{\mathrm{P}}^{s}\right)^{2}\right)}{D^{s}}, \\
& D^{s}=1-2 v_{\mathrm{P}}^{s} v_{\mathrm{TP}}^{s} v_{\mathrm{PT}}^{s}-2 v_{\mathrm{TP}}^{s} v_{\mathrm{PT}}^{s}-\left(v_{\mathrm{P}}^{s}\right)^{2}, \\
& \beta_{\mathrm{P}}^{s}=A_{\mathrm{P}}^{s} \alpha_{\mathrm{P}}^{s}+A_{\mathrm{PT}}^{s} \alpha_{\mathrm{P}}^{s}+A_{\mathrm{TP}}^{s} \alpha_{\mathrm{T}}^{s}, \\
& \beta_{\mathrm{T}}^{s}=2 A_{\mathrm{TP}}^{s} \alpha_{\mathrm{P}}^{s}+A_{\mathrm{T}}^{s} \alpha_{\mathrm{T}}^{s}, v_{\mathrm{TP}}^{s} E_{\mathrm{P}}^{s}=v_{\mathrm{PT}}^{s} E_{\mathrm{T}}^{s}
\end{aligned}
$$

The mechanical response of the TM model is to be evaluated considering the temperature field change that results as a steady-state solution to the heat transfer BVP with the imposed temperature difference $\Delta T_{i}^{I M}=\Delta T_{i}$, $\Delta T_{i}^{A}=\Delta T_{o} \quad$ and $\quad \Delta T_{o}^{I M}=\Delta T_{i}^{A}=\Delta T_{m}, \quad$ applied respectively on the inner $\left(\rho_{i}^{I M}\right)$ and outer $\left(\rho_{o}^{A}\right)$ surface of the TM model and on the interface $\left(\rho_{o}^{I M}=\rho_{i}^{A}\right)$ surface between the two layers. Since the TM model is a fictitious model, isotropic and temperature independent thermal properties can be assumed, which yields the following temperature distribution:

$$
T^{s}(r)=\Delta T_{i}^{s}+\frac{\Delta T_{o}^{s}-\Delta T_{i}^{s}}{\ln \left(\frac{\rho_{o}^{s}}{\rho_{i}^{s}}\right)} \ln \left(\frac{r}{\rho_{i}^{s}}\right) ; \rho_{i}^{s} \leq r \leq \rho_{o}^{s}
$$

The displacement field $u^{s}(r)$ can be determined as a solution of the two pairs of first-order differential equations establishing the relationship between the variables $u^{s}(r)$ and $\sigma_{r r}^{s}(r)$ [17]: 


$$
\begin{aligned}
& \frac{\mathrm{d} u^{s}}{\mathrm{~d} r}=-\frac{A_{\mathrm{PT}}^{s}}{A_{\mathrm{P}}^{s}} \frac{u^{s}}{r}+\frac{\sigma_{r r}^{s}}{A_{\mathrm{P}}^{s}}+\frac{\beta_{\mathrm{P}}^{s}}{A_{\mathrm{P}}^{s}} T^{s} \\
& \frac{\mathrm{d} \sigma_{r r}^{s}}{\mathrm{~d} r}=\left(A_{\mathrm{P}}^{s}-\frac{\left(A_{\mathrm{PT}}^{s}\right)^{2}}{A_{\mathrm{P}}^{s}}\right) \frac{u^{s}}{r^{2}}+\left(\frac{A_{\mathrm{PT}}^{s}}{A_{\mathrm{P}}^{s}}-1\right) \frac{\sigma_{r r}^{s}}{r}+ \\
& +\left(\frac{A_{\mathrm{PT}}^{s}}{A_{\mathrm{P}}^{s}}-1\right) \frac{\beta_{\mathrm{P}}^{s} T^{s}}{r} ; \quad \rho_{i}^{s} \leq r \leq \rho_{o}^{s}
\end{aligned}
$$

subject to the traction-free boundary conditions:

$$
\sigma_{r r}^{I M}\left(\rho_{i}^{I M}\right)=0, \quad \sigma_{r r}^{A}\left(\rho_{o}^{A}\right)=0
$$

and the continuity conditions at the layers' interface:

$$
\sigma_{r r}^{I M}\left(\rho_{o}^{I M}\right)=\sigma_{r r}^{A}\left(\rho_{i}^{A}\right), \quad u_{r}^{I M}\left(\rho_{o}^{I M}\right)=u_{r}^{A}\left(\rho_{i}^{A}\right)
$$

Finally, by a proper consistent combination of the TM model parameters the required stress and configuration correspondence between the $\Psi_{1 \lambda}^{\mathrm{C}}$ and $\Omega_{1 \lambda}^{\mathrm{C}}$ states is established. With this, all the necessary data required in the subsequent analysis of the real arterial segment (sub-steps 3.2) are obtained.

\section{Case study - Treating a patient-specific arterial segment}

Similarly as discussed in the beam example, the only data that are available when treating a patient-specific artery are its loaded (in vivo) configuration $\Omega_{2}$ under the exerted blood pressure variation. In the sequel, the solution methodology based on the TMA approach will be used to determine the in vivo stress state of a patientspecific arterial segment. In the case study, experimental

a

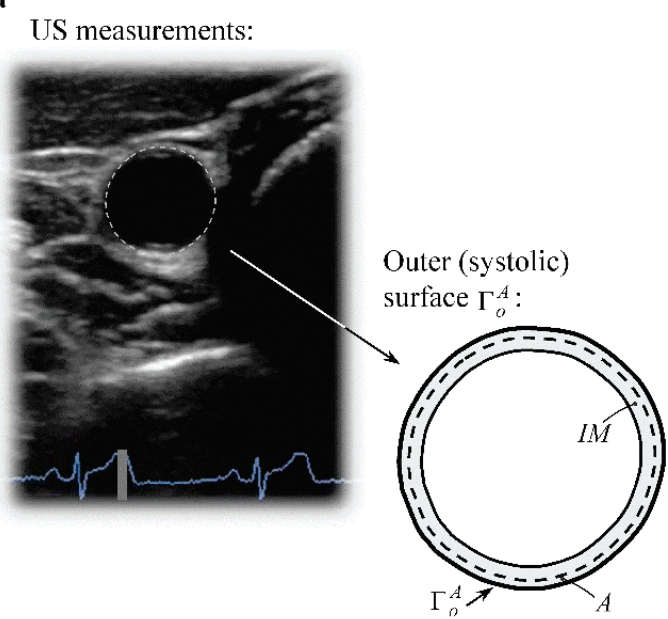

data of a healthy, non-smoking 28-year-old male's CCA are considered.

\subsection{Experimental data}

The CCA in vivo configuration was obtained using a high-resolution echo-tracking device Philips iU22 (Philips Healthcare) and a L9-3 linear probe. Ultrasound (US) image of the CCA transversal view (in systole) was recorded approximately $20 \mathrm{~mm}$ proximal to the carotid bulb. From the US image, the outer contour of the adventitia layer $\left(\Gamma_{o}^{A}\right)$ was then obtained, Fig. 5a. The average value of the corresponding arterial thickness $\delta_{s y s}$ was obtained using M-mode US measurements (Fig. 5b), being $0.45 \mathrm{~mm}$. The in vivo cross section of the CCA configuration, depicted in Fig. 5a, was then obtained by assuming the $I M$ thickness represents two-thirds and the $A$ thickness one-third of the total arterial thickness [2, 21]. For the purpose of numerical analysis, the arterial segment was assumed to be straight in the longitudinal direction.

An average distention waveform cycle of the inner arterial radius $r_{i}^{I M}(t)$ was obtained from M-mode US measurements (Fig. 5b) by averaging the distention waveforms of three heart cycles. During US measurements, the blood pressure was measured on the subject's left brachial artery. The pressure-radius waveform of the CCA segment (Fig. 5b) was then obtained by, first, assuming that the mean and diastolic pressures are nearly constant throughout the large artery tree [22] and, then, by combining the diameter-derived pulse pressure method [23] and the exponential relationship between the arterial cross-sectional area and pressure, as presented in [24, 25].

b

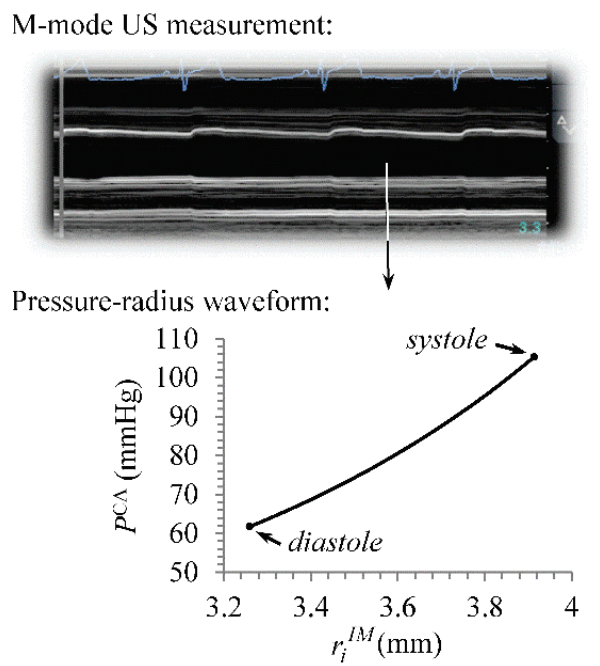

Figure 5 Experimental data of the CCA segment: (a) the in vivo CCA geometry (where $\Gamma_{o}^{A}$ is the outer adventitia surface in systole, and $I M$ and $A$ are the intima-media and adventitia layers) and (b) the obtained blood-pressure waveform

\subsection{Arterial behaviour characterization}

In characterizing the arterial behaviour we make use of the equivalent circular arterial segment, as discussed in previous sections. Arterial material properties can be either presumed from literature or can be identified from the measured pressure-dilatation waveform through the heart cycle of an artery. The feasibility of characterizing 
the arterial material properties based on the in vivo clinical data was, for instance, demonstrated by SchulzeBauer and Holzapfel [21], Masson et al. [26] and Stålhand et al. [27]. In order to assess the stress state in the circular arterial segment, and consequently in the real artery, a recourse to certain less or more objective suppositions and in vitro obtained data is needed. Consequently, it is on the degree of truthfulness of the adopted unknown information that the objectivity of the computed stress depends.

Regarding stretching of the arterial segment the following two suppositions were adopted in the investigated case: i) the in vivo axial stretch $\lambda$ was set to $10 \%$ (obtained based on measurements made in situ, before extraction of the human carotids [9]), and ii) the axial contraction $\Lambda$, which is associated with the longitudinal cut of the unloaded arterial segment, is set to unity [26]. With these stretches assumed the respective lengths of the arterial segment in the unloaded configuration $\Omega_{1}^{\mathrm{C}}$ and zero-stress configuration $\Omega_{0}^{\mathrm{C}}$ are specified as well.

As a principal constraint imposed in determining the mechanical response of a circular arterial segment is the acceptance of the uniform strain hypothesis [7], which presumes a uniform transmural distribution of circumferential strain at physiological conditions. Besides that, we assume that some additional knowledge relating to the stress distribution is available. Explicitly, we assume: i) the ratio $\kappa_{1}$ between the circumferential and axial stress is known for one (arbitrary) value of the blood pressure, and ii) the ratio $\kappa_{2}$ between the circumferential stresses at the $I M-A$ interface is known as well. In this work, according to Masson et al. [28], where CCA circular segments in seven young subjects were characterized, $\kappa_{1}$ was set to 1.9 (corresponding to the segment's outer media surface in systole), and $\kappa_{2}$ was set to 5.0, according to [29].

The characterization of the circular arterial segment's behaviour is twofold; it encompasses identification of the zero-stress configuration $\Omega_{0}^{\mathrm{C}}$ parameters (and $\Theta_{0}$ ), on one hand, and complete material parameters characterisation $\left(c_{a}^{A}, c_{b}^{A}, \quad c_{a}^{I M}\right.$ and $c_{b}^{I M}$ for the assumed constitutive behaviour), on the other hand. This inverse problem can be resolved by solving a minimization problem. The goal is to obtain such values of the unknown parameters $\boldsymbol{u}=\left\{R_{o}^{A}, \Theta_{0}, c_{a}^{A}, c_{b}^{A}, c_{a}^{I M}, c_{b}^{I M}\right\}$, which will match the computed response of the circular arterial segment to the experimental response as close as possible. The measure of the agreement is given by the objective function $\chi=\chi(\boldsymbol{u})$, formulated as:

$$
\begin{aligned}
& \chi(\boldsymbol{u})=\chi_{\mathrm{P}}(\boldsymbol{u})+\chi_{\lambda}(\boldsymbol{u})=\sum_{k=1}^{N_{P}}\left(P_{k}(\boldsymbol{u})-P_{k}^{\mathrm{CA}}\right)^{2}+ \\
& +g_{\lambda}\left(\lambda_{\phi}^{i}(\boldsymbol{u})-\lambda_{\phi}^{o}(\boldsymbol{u})\right)^{2}
\end{aligned}
$$

with functions $\chi_{\mathrm{P}}(\boldsymbol{u})$ and $\chi_{\lambda}(\boldsymbol{u})$ accounting for: i) the difference between the computed and experimental pressure load, represented respectively by the computed and measured intraluminal pressures $P_{k}(\boldsymbol{u})$ and $P_{k}^{\mathrm{CA}}$, and ii) the transmural gradient of the circumferential strain, taking into account the uniform strain hypothesis. In relation (19), $N_{P}$ denotes the number of points from the experimentally obtained pressure-radius waveform (Fig. 5b) considered in the optimization, $\lambda_{\phi}^{i}=\lambda_{\phi}^{I M}\left(r_{i}^{I M}\right)$ and $\lambda_{\phi}^{o}=\lambda_{\phi}^{A}\left(r_{o}^{A}\right)$ are the computed circumferential stretches at the inner and outer surface of the segment's wall, and $g_{\lambda}$ is the weighting factor.

With the obtained best-fit values, presented in Table 1 , the characterization of the circular arterial segment is accomplished. In the following, in order to apply the TMA approach, we are interested in the $\Omega_{1 \lambda}^{\mathrm{C}}$ state of the arterial segment. As already discussed, based on this state the associated TM model is characterized, as performed in the following subsection.

Table 1 The identified parameters of the circular arterial segment

\begin{tabular}{|c|c|c|c|c|c|}
\hline$R_{o}^{A}(\mathrm{~mm})$ & $\Theta_{0}\left({ }^{\circ}\right)$ & $c_{a}^{A}(\mathrm{kPa})$ & $c_{b}^{A}$ & $c_{a}^{I M}(\mathrm{kPa})$ & $c_{b}^{I M}$ \\
\hline 4,93 & 117,96 & 30,47 & 0,106 & 80,07 & 1,13 \\
\hline
\end{tabular}

\subsection{The TM model characterization}

In order to apply the TMA approach to the observed patient-specific arterial geometry, the properties of the TM model need to be properly defined first. Considering that the mathematical framework governing the mechanical response of the TM model is given by the Eqs. (13) - (18), the characterization actually means finding out such values of the TM model parameters with which the correspondence between the stress and configuration of states $\Psi_{1 \lambda}^{\mathrm{C}}$ and $\Omega_{1 \lambda}^{\mathrm{C}}$ will be established. Note that the targeting state $\Omega_{1 \lambda}^{\mathrm{C}}$ has been determined in the previous section.

The zero-stress configuration $\Psi_{0}^{\mathrm{C}}$ of the two-layered TM model is defined based on the loaded (systolic) geometry of the corresponding idealized arterial segment (configuration $\Omega_{2}^{\mathrm{C}}$, Fig. 4b). More specifically, the geometry $\Gamma_{2}^{\mathrm{C}}$ is adopted as the interface surface between the layers of the TM model, whereas the corresponding layer thicknesses $\delta_{\psi}^{I M}$ and $\delta_{\psi}^{A}$ (Fig. 4c) are initially unknown and have to be properly determined within the TM model characterisation procedure. Although it could be realised differently, the choice of relating the TM model's interface surface with the geometry $\Gamma_{2}^{\mathrm{C}}$ is considered to give the TM model the best configuration correspondence and robustness for the subsequent application to the real artery.

The TM model characterisation includes: i) determination of the thermal loadings 
$\left(\Delta T_{i}^{I M}=\Delta T_{i}, \Delta T_{i}^{A}=\Delta T_{o}\right.$ and

$\left.\Delta T_{o}^{I M}=\Delta T_{i}^{A}=\Delta T_{m}\right)$, ii) characterization of material parameters (being $E_{\mathrm{P}}^{\psi I M}, E_{\mathrm{T}}^{\psi I M}, E_{\mathrm{P}}^{\psi A}, E_{\mathrm{T}}^{\psi A}$, $\alpha_{\mathrm{P}}^{\psi I M}, \alpha_{\mathrm{T}}^{\psi I M}, \alpha_{\mathrm{P}}^{\psi A}, \alpha_{\mathrm{T}}^{\psi A} ;$ where the Poisson ratios are assumed given: $\quad v_{\mathrm{P}}^{\psi I M}=v_{\mathrm{P}}^{\psi A}=0,45 \quad$ and $\left.v_{\mathrm{PT}}^{\psi I M}=v_{\mathrm{PT}}^{\psi A}=0,1\right)$, and iii) determination of the respective layer thicknesses $\left(\delta_{\psi}^{I M}\right.$ and $\left.\delta_{\psi}^{A}\right)$. The characterisation is treated as an optimisation problem with the TM model calculations performed considering different values for the open model parameters. It is supposed, accordingly, that the values of the optimisation variables' vector $\boldsymbol{v}$ :

$$
\begin{aligned}
\boldsymbol{v}= & \left(\Delta T_{i}, \Delta T_{m}, \Delta T_{o}, E_{\mathrm{P}}^{\psi I M}, E_{\mathrm{T}}^{\psi I M}, E_{\mathrm{P}}^{\psi A}, E_{\mathrm{T}}^{\psi A}, \alpha_{\mathrm{P}}^{\psi I M},\right. \\
& \left., \alpha_{\mathrm{T}}^{\psi I M}, \alpha_{\mathrm{P}}^{\psi A}, \alpha_{\mathrm{T}}^{\psi A}, \delta_{\psi}^{I M}, \alpha_{\psi}^{A}\right)
\end{aligned}
$$

obtained by the minimisation of the corresponding objective function $\chi=\chi(v)$, give the required correspondence between the $\Psi_{1 \lambda}^{\mathrm{C}}$ and $\Omega_{1 \lambda}^{\mathrm{C}}$ state. In order to account for: i) the relative error between the computed $\Psi_{1 \lambda}^{\mathrm{C}}$ and targeted $\Omega_{1 \lambda}^{\mathrm{C}}$ stress states, and ii) the discrepancy between the computed geometry of the TM model and the geometry of the circular arterial segment in the configuration $\Omega_{1 \lambda}^{\mathrm{C}}$, the objective function $\chi=\chi(v)$ is structured as follows:

$$
\begin{aligned}
& \chi(\boldsymbol{v})=\sum_{m=r, \phi, z} \frac{1}{N_{m}} \sum_{k=1}^{N_{m}}\left(\frac{S_{\mathrm{c} k}^{m}(\boldsymbol{v})-S_{k}^{m}}{S_{k}^{m}}\right)^{2}+ \\
& +g_{\rho} \sum_{k=i, m, o}\left(\frac{\rho_{k}^{\Psi}(v)-\rho_{k}}{\rho_{k}}\right)^{2}
\end{aligned}
$$

where the vectors $\boldsymbol{S}_{\mathrm{c}}^{m}$ and $\boldsymbol{S}^{m} ; m \in\{r, \phi, z\}$ are holding respectively the computed $\left(\Psi_{1 \lambda}^{\mathrm{C}}\right)$ and targeted $\left(\Omega_{1 \lambda}^{\mathrm{C}}\right)$ discrete values of the radial, circumferential, and axial stress $^{2}$, and $N_{m} ; m \in\{r, \phi, z\}$ is the length of the vector $\boldsymbol{S}^{m}$. The quantities $\rho_{k}^{\Psi}$ and $\rho_{k} ; k \in\{r, \phi, z\}$ belong to the computed $\Psi_{1 \lambda}^{\mathrm{C}}$ and targeted $\Omega_{1 \lambda}^{\mathrm{C}}$ state, respectively, and represent the inner $(i)$, interfacial $(m)$ and outer $(o)$ radii. The scalar $g_{\rho}$ is the weighting factor.

The minimisation of the objective function $\chi=\chi(v)$ yields the best-fit values of the TM model parameters, tabulated in Tab. 2. The accomplished identification is a significant step in the TMA approach, because the identified values of the geometrical and

\footnotetext{
${ }^{2}$ Note that in $\boldsymbol{S}^{m}$, only the non-zero values of the stresses are included.
}

material parameters also define the CCA TM model $\Psi_{0}$, according to the TMA approach. By applying the identified thermal loads onto the CCA TM model, its loaded configuration $\Psi_{1 \lambda}$ follows.

Table 2 The identified parameters of the circular TM segmen

\begin{tabular}{|c|c|c|c|c|c|}
\hline Layer $(\mathrm{s})$ & $\begin{array}{l}E_{P}^{\psi s} \\
(\mathrm{kPa})\end{array}$ & $\begin{array}{l}\alpha_{P}^{\psi s} \\
(1 / \mathrm{K})\end{array}$ & $\begin{array}{l}E_{T}^{\psi s} \\
(\mathrm{kPa})\end{array}$ & $\begin{array}{l}\alpha_{T}^{\psi s} \\
(1 / \mathrm{K})\end{array}$ & $\begin{array}{l}\delta_{\psi}^{s} \\
(\mathrm{~mm})\end{array}$ \\
\hline$I M$ & 240,734 & $-0,275$ & 407,604 & $-0,055$ & 0,630 \\
\hline$A$ & 138,012 & $-0,270$ & 166,909 & $-0,051$ & 0,305 \\
\hline & $\Delta T_{i}$ & $\Delta T_{m}$ & $\Delta T_{o}$ & & \\
& $\left({ }^{\circ} \mathrm{C}\right)$ & $\left({ }^{\circ} \mathrm{C}\right)$ & $\left({ }^{\circ} \mathrm{C}\right)$ & & \\
\hline & 0,954 & 1,181 & 1,254 & & \\
\hline
\end{tabular}

\subsection{Results and discussion}

In this section, the results of stress determination of the observed patient-specific CCA are presented.

The purpose of considering the possibility of computing the RSs by means of the corresponding TM model is not at all a demonstration of an alternative solution way, as one could conclude from the study in the previous section, but exclusively exposing the potential the TMA approach possesses in regard to the treatment of the real arteries' geometries. Since the above specified TM model $\Psi_{0}^{\mathrm{C}}$ gives correct distribution of RSs and geometry of the stretched configuration $\Omega_{1 \lambda}^{\mathrm{C}}=\Psi_{1 \lambda}^{\mathrm{C}}$ in the case of the idealized arterial segment by definition, the computed stresses in the loaded configuration $\Omega_{2}^{\mathrm{C}}$, when the blood pressure is applied, are correct as well. If now, instead of on the geometry $\Gamma_{2}^{\mathrm{C}}$ (Fig. 4b) the geometry $\Gamma_{o}^{A}$ of the real arterial segment (Fig. 5a) is taken as the interface surface between the layers, and the above identified thicknesses $\delta_{\psi}^{I M}$ and $\delta_{\psi}^{A}$ are assumed as the respective layer thicknesses, a new TM model $\Psi_{0}$ is obtained. The material behaviour and temperature loads of the model are also taken from the circular TM model. In analogy with the proven response behaviour of the $\Psi_{0}^{\mathrm{C}}$ TM model, it is expected that the $\Psi_{0}$ TM model is capable to give a reasonably fair approximation of the distribution of RSs and geometry of the stretched configuration $\Omega_{1 \lambda} \approx \Psi_{1 \lambda}$ of the real arterial segment, which is a precondition for the reliability of the subsequent stress state determination in the loaded configuration $\Omega_{2}$ of the artery.

The stresses in the patient-specific artery are thus obtained in two steps. Firstly, the distribution of RSs is computed by means of the $\Psi_{0}$ TM model (sub-step 3.2). Secondly, based on the obtained RSs and configuration $\Omega_{1 \lambda} \approx \Psi_{1 \lambda}$ the arterial segment is exposed to the actual blood pressure variation (sub-step 3.3). 


\subsubsection{Prediction of the CCA stretched state}

While the computation of the mechanical response of the $\Psi_{0}^{\mathrm{C}}$ TM model could be done analytically, due to rotational symmetry of the idealized arterial segment, the analysis of the $\Psi_{0}$ TM model has to be performed numerically. The most convenient method to cope with the irregular 3D geometry of the CCA is the finite element (FE) method. The volume domain representing the TM model was discretized into approximately 20000 finite elements with temperature and displacement degrees of freedom (Fig. 6).

For the TM model's material mechanical behaviour the values in Tab. 2 are adopted, while for the heat transfer analysis the choice of the assumed isotropic and temperature independent thermal properties is arbitrary, because of the steady-state solution and prescribed temperatures as the boundary conditions.

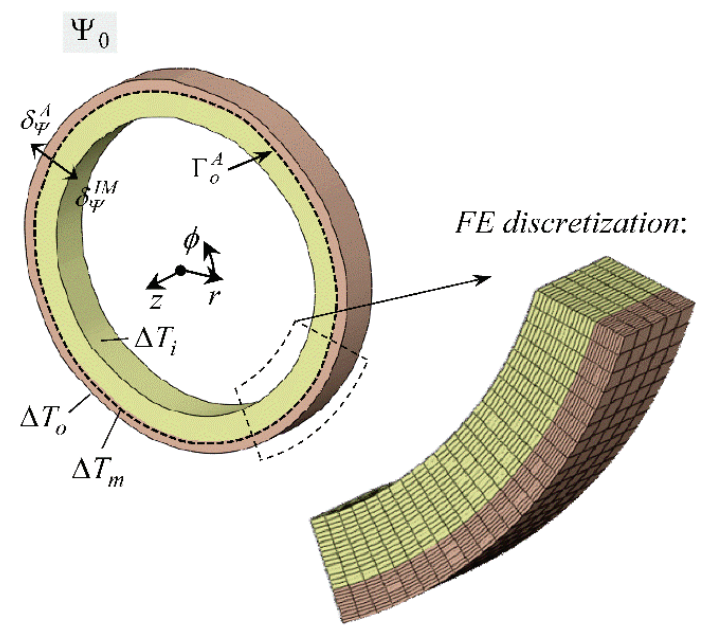

Figure 6 The Geometrical model of the TM arterial model $\left(\Psi_{0}\right)$ and a detail of the FE discretization

In computing the mechanical response of the TM model, first, the steady-state heat conduction problem is solved. The resulting temperature distribution over the thickness of the segment is obtained based on the applied thermal loadings ( $\Delta T_{i}, \Delta T_{o}$ and $\Delta T_{m}$ ), identified for the circular TM segment $\Psi_{0}^{\mathrm{C}}$. The established temperature field gives rise to the stress and displacement field distributions in the TM model, which approximate the RSs and geometry of the arterial segment in its stretched configuration $\Omega_{1 \lambda}$.

In Fig. 7a, both the geometry of the $\Omega_{1 \lambda}$ configuration and the residual circumferential stress distribution are displayed. The bending-like distribution through the arterial wall thickness is easily recognizable, with the circumferential stress in the $I M$ layer ranging from $-9 \mathrm{kPa}$ on the inner surface to approx. $5 \mathrm{kPa}$ on the outer surface, whereas in the $A$ layer, in contrast to $I M$, but fully in accordance with the bending nature, the stress is tensile, with an average value around $4 \mathrm{kPa}$. The residual circumferential and axial stress distributions through the wall thickness along four $90^{\circ}$ sector's lines $\left(L^{0}, L^{90}, L^{180}, L^{270}\right)$ are plotted in Fig. $7 \mathrm{~b}$, where for comparison also the RSs of the circular arterial segment are displayed. Through the wall thickness the axial stresses practically coincide, whereas the circumferential stresses differ slightly. On one hand, this could be attributed to the CCA somewhat irregular geometry, and on the other hand, to the FE discretization (which, especially, has an effect near the inner and outer surface of the $I M$ layer). Irrespective of that, the predicted RSs are in quantitative agreement with the data from prior works in this field $[13,30]$.

In Fig. $7 b$, additionally, the stresses predicted with the circular TM segment ( $\Psi_{1 \lambda}^{\mathrm{C}}$ state) are presented. As seen in the figure, the stress distribution of the TM segment practically overlaps the stresses of the circular arterial segment ( $\Omega_{1 \lambda}^{\mathrm{C}}$ state). This was expected since the properties of the TM model are determined based on the $\Omega_{1 \lambda}^{\mathrm{C}}$ state stresses and configuration. However, what this result shows is the feasibility of using the TMA approach for predicting the arterial bending as well as stretching stresses.

\subsubsection{The predicted CCA loaded state}

The deformed state $\Psi_{1 \lambda}$ of the CCA TM model is supposed to resemble the stretched configuration $\Omega_{1 \lambda}$ of the CCA. Considering further that in the $\Psi_{1 \lambda}$ configuration the stresses of the TM model approximate the RSs in the $\Omega_{1 \lambda}$ configuration, the deformed and stressed $\Psi_{1 \lambda}$ FE model can be adopted as the startingpoint for the subsequent analyses regarding the arterial mechanical response. Although the zero-stress configuration $\Omega_{0}$ is not known, the strains in the $\Omega_{1 \lambda}$ configuration can be determined by assigning the assumed arterial material behaviour to the adopted stressed FE model, which completes determination of the $\Omega_{1 \lambda}$ state.

The loaded state $\Omega_{2}$ of the artery is obtained by exposing the obtained FE model, representing the $\Omega_{1 \lambda}$ state, to the applied blood pressure. Both the geometry of the $\Omega_{2}$ configuration and the circumferential stress distribution, predicted for the CCA loaded state (in systole), are displayed in Fig. 8a. As seen in the figure, the stresses are dominant in the $I M$ layer, with the peak value around $200 \mathrm{kPa}$, whereas the average stress value in the $A$ layer is around 40 to $50 \mathrm{kPa}$, which is in quantitative agreement with the data from [29]. In Fig. 8b, the circumferential and axial stress distributions through the wall thickness along four $90^{\circ}$ sector's lines $\left(L^{0}, L^{90}\right.$, $\left.L^{180}, L^{270}\right)$ are plotted. In comparison to the circumferential stress distribution, predicted for the loaded circular arterial segment, the circumferential stresses of the CCA are somewhat higher. On one hand, this occurs as a consequence of compressive RSs, which are in the case of the CCA slightly lower at the inner IM surface. Another reason is the variable thickness of the CCA stretched state $\Omega_{1 \lambda}$, which affects the stresses, caused by the application of the blood pressure, notably. 


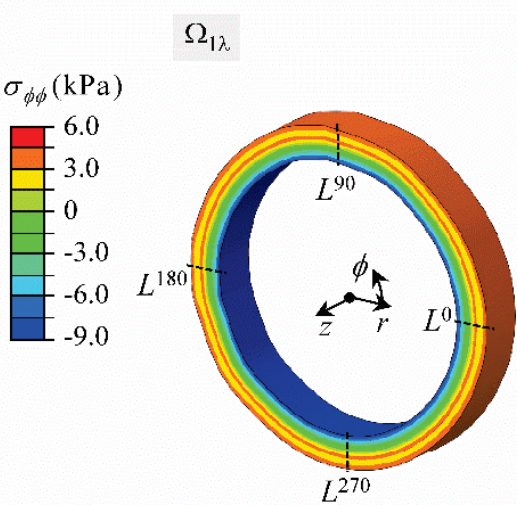

b

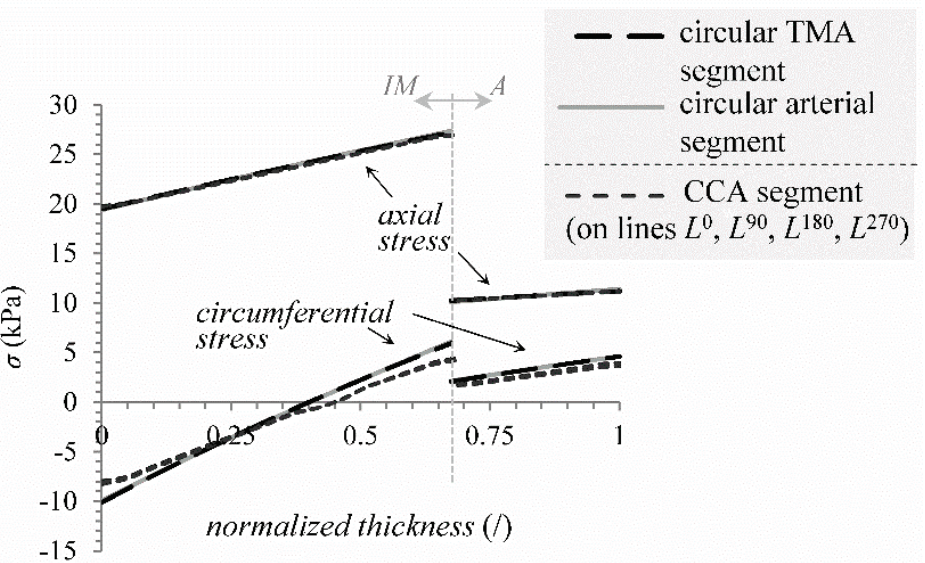

Figure 7 The predicted stretched states $\left(\Omega_{1 \lambda}\right)$ : (a) residual circumferential stresses of the CCA segment and (b) stresses predicted for the CCA, circular arterial and circular TMA segment

a

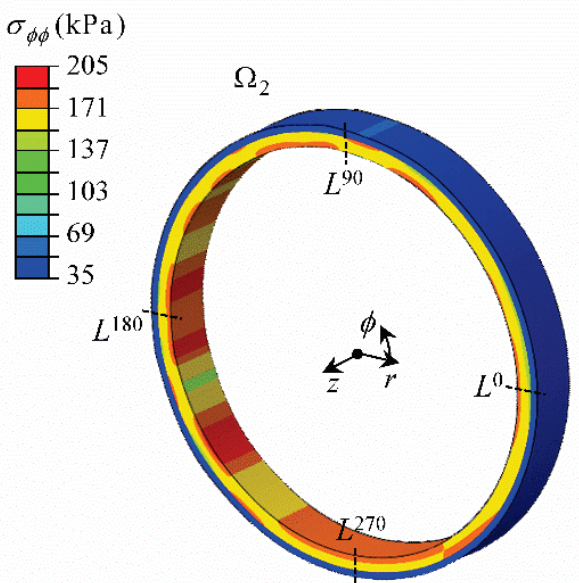

b

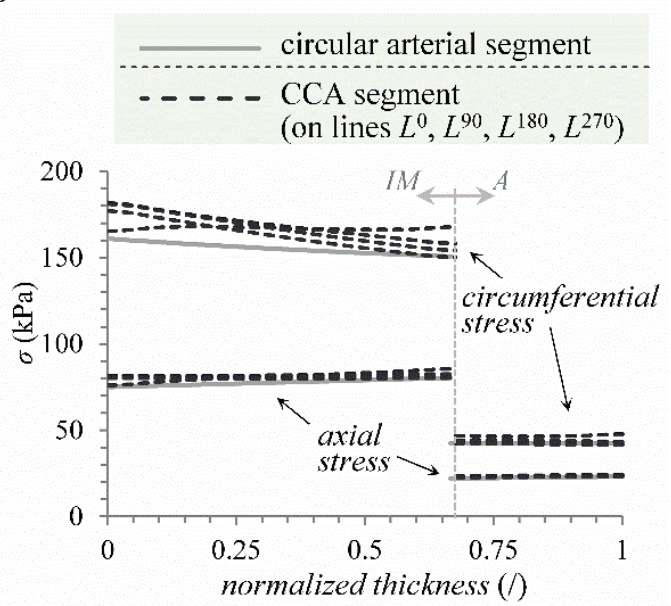

Figure 8 The predicted loaded (systolic) states $\left(\Omega_{2}\right)$ : (a) circumferential stresses of the CCA and (b) stresses predicted for the CCA and circular arterial segment

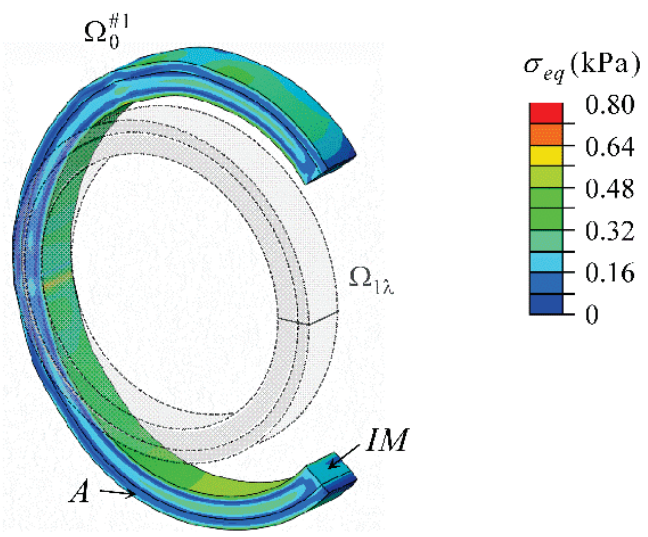

b

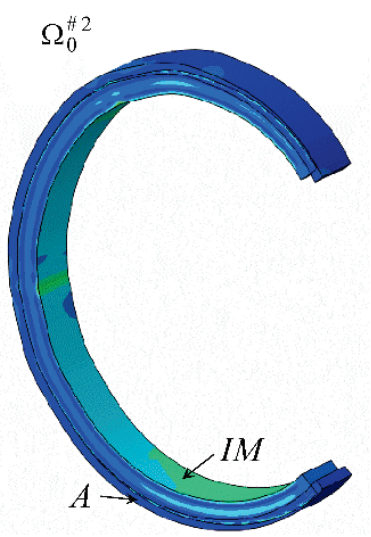

Figure 9 Predictions of the CCA zero-stress state: (a) the CCA cut-open state $\Omega_{0}^{\# 1}$, and (b) the CCA cut-open state with both layers further delaminated

\subsubsection{Validation of the TMA approach}

The TMA approach is validated by evaluating the stresses in the presumed CCA zero-stress state $\Omega_{0}$. Accordingly, the CCA in the $\Omega_{1 \lambda}$ state is cut twice: by the first, transversal, cut the in vivo stretch $\lambda$ is released, while by the second, longitudinal, cut the arterial segment cutopens (see Fig. 4a).

The predicted equilibrium state of the arterial segment, obtained by transversally and longitudinally cutting the CCA from the $\Omega_{1 \lambda}$ state, is represented by the von Mises equivalent stress, displayed in Fig. 9a. As seen in the figure, the obtained state is almost free of RSs. In this state, however, both layers are still considered tight together. If now the two layers were further delaminated, the stresses in the separated layers would even further reduce, as shown in Fig. 9b. Considering that for the circular arterial segment the same opening angle was assumed for both layers, we could expect the same would occur in the case of CCA. However, because of its 
irregular geometry, slightly different amount of openings is predicted.

\section{Conclusion}

An important aspect in modelling the stress state of human arteries is the inclusion of RSs. Besides having a significant influence on the in vivo stress distribution, understanding of RSs seems to be crucial in consideration of vascular growth and remodelling. Although several methods can be found in literature to include RSs in human (i.e., patient-specific) arteries, none of them is universally accepted. They are mostly very time consuming and are able to predict only the arterial RSs, whereas they cannot predict the corresponding geometry of the artery, or the predicted arterial state is not fully consistent.

The main purpose of this work is to prove the potential of the TMA approach in predicting the in vivo stresses in the human CCA segment, with the arterial RSs accounted for. As demonstrated, the approach enables to predict the patient-specific arterial residual stress state (and the corresponding configuration) by means of an auxiliary TM model and solving the related BVP. Because the stress-free configuration of the TM model is based on the in vivo geometry of the artery, there is no need at all to model the unknown CCA cut-open zero-stress configuration. Although in this work the CCA was treated as a twolayered structure of constant cross-section, the approach can be used as well to account for all three arterial layers and axially variable cross-section. Also, in principle, there is no restriction in its applicability regarding consideration of another material behaviour law.

The real benefit of the TMA approach can be regarded from the point of the arterial residual stress state modelling. Without the TMA approach, normally, several repetitions of numerical analysis are needed to find the proper residual stress state [4], which can be very time consuming. In the case of the TMA approach, on the contrary, applying the material properties of the circular TM model and respective thermal loads to the stress-free in vivo CCA geometry yields an approximation to the CCA residual stress state in one single numerical analysis. Furthermore, the approach also enables a prediction of the residual stress state configuration, which can be considered as a major drawback of most existing methods. Although they predict the RSs, they cannot predict the corresponding geometry of the residual stress state configuration.

In the end, considering the numerical efficiency and all the properties of the TMA approach, discussed in detail in this work, a conclusion can be drawn that the approach could contribute, along with the non-invasive medical imaging techniques, to better assessment and understanding of the in vivo stress state of patient-specific arteries.

\section{References}

[1] Jiang, Y.; K. Kohara; K. Hiwada Association Between Risk Factors for Atherosclerosis and Mechanical Forces in Carotid Artery. // Stroke. 31, 10(2000), pp. 2319-2324. https://doi.org/10.1161/01.STR.31.10.2319

[2] Holzapfel, G. A.; Gasser, T. C.; Ogden, R. W. Comparison of a multi-layer structural model for arterial walls with a fung-type model, and issues of material stability. // J Biomech Eng. 126, 2(2004), pp. 264-275. https://doi.org/10.1115/1.1695572

[3] Cardamone, L. et al. Origin of axial prestretch and residual stress in arteries. // Biomech Model Mechanobiol. 8, 6(2009), pp. 431-446. https://doi.org/10.1007/s10237-008-0146-x

[4] Polzer, B. et al. A numerical implementation to predict residual strains from the homogeneous stress hypothesis with application to abdominal aortic aneurysms. // Annals of Biomedical Engineering. 41, 7(2013), pp. 1516-1527. https://doi.org/10.1007/s10439-013-0749-y

[5] Kassab, G. S. Biomechanics of the cardiovascular system: the aorta as an illustratory example. // Journal of the Royal Society Interface. 3, 11(2006), pp. 719-740. https://doi.org/10.1098/rsif.2006.0138

[6] Chuong, C. J.; Fung, Y. C. On Residual-Stresses in Arteries. // Journal of Biomechanical EngineeringTransactions of the Asme. 108, 2(1986), pp. 189-192. https://doi.org/10.1115/1.3138600

[7] Takamizawa, K.; Hayashi, K. Strain energy density function and uniform strain hypothesis for arterial mechanics. // J Biomech. 20, 1(1987), pp. 7-17. https://doi.org/10.1016/0021-9290(87)90262-4

[8] Wang, R.; Gleason, R. L. A mechanical analysis of conduit arteries accounting for longitudinal residual strains. // Annals of Biomedical Engineering. 38, 4(2010), pp. 13771387. https://doi.org/10.1007/s10439-010-9916-6

[9] Delfino, A., et al. Residual strain effects on the stress field in a thick wall finite element model of the human carotid bifurcation // Journal of Biomechanics, 30, 8 (1997), pp. 777-786. https://doi.org/10.1016/S0021-9290(97)00025-0

[10] Bustamante, R.; Holzapfel, G. A. Methods to compute 3D residual stress distributions in hyperelastic tubes with application to arterial walls. // International Journal of Engineering Science. 48, (2010), pp. 1066-1082. https://doi.org/10.1016/j.ijengsci.2010.06.005

[11] Gee, M. W.; Förster, Ch.; Wall, W. A. A computational strategy for prestressing patient-specific biomechanical problems under finite deformation. // International Journal for Numerical Methods in Engineering. 26, (2010), pp. 5272. https://doi.org/10.1002/cnm.1236

[12] Lu, J.; Zhou, X.; Raghavan, M. L. Inverse method of stress analysis for cerebral aneurysms. // Biomech Model Mechanobiol. 7, 6(2008), pp. 477-486. https://doi.org/10.1007/s10237-007-0110-1

[13] Alastrué, V. et al. Assessing the use of the "opening angle method" to enforce residual stresses in patient-specific arteries. // Annals of Biomedical Engineering. 35, 10(2007), pp. 1821-1837. https://doi.org/10.1007/s10439-007-9352-4

[14] Schroder, J.; Brinkhues, S. A novel scheme for the approximation of residual stresses in arterial walls. // Archive of Applied Mechanics. 84, 6(2014), pp. 881-898. https://doi.org/10.1007/s00419-014-0838-x

[15] Alastrue, V. et al. Anisotropic micro-sphere-based finite elasticity applied to blood vessel modelling. // Journal of the Mechanics and Physics of Solids. 57, 1(2009), pp. 178203. https://doi.org/10.1016/j.jmps.2008.09.005

[16] Alford, P. W.; Humphrey, J. D.; Taber, L. A. Growth and remodeling in a thick-walled artery model: effects of spatial variations in wall constituents. // Biomechanics and Modeling in Mechanobiology. 7, 4(2008), pp. 245-262. https://doi.org/10.1007/s10237-007-0101-2

[17] Urevc, J. et al. Applying Thermomechanical Analogy to Predict the Arterial Residual Stress State. // Strojniški vestnik - Journal of Mechanical Engineering. 61, 1(2015), pp. 5-23. https://doi.org/10.5545/sv-jme.2014.2061

[18] Stålhand, J. Determination of human arterial wall parameters from clinical data. // Biomech Model Mechanobiol. 8, (2009), pp. 141-148. https://doi.org/10.1007/s10237-008-0124-3 
[19] Humphrey, J. D.; Na, S. Elastodynamics and arterial wall stress. // Annals of Biomedical Engineering. 30, (2002), pp. 509-523. https://doi.org/10.1114/1.1467676

[20] Takamizawa, K.; Nakayama, Y. Stress distribution in a bilayer elastic model of a coronary artery. // Journal of Applied Mechanics. 80, (2013), pp. 041006,1-041006,6.

[21] Schulze-Bauer, C. A. J.; Holzapfel, G. A. Determination of constitutive equations for human arteries from clinical data. // Journal of Biomechanics. 36, (2003), pp. 165-169. https://doi.org/10.1016/S0021-9290(02)00367-6

[22] Verbeke, F. et al. Noninvasive assessment of local pulse pressure: importance of brachial-to-radial pressure amplification. // Hypertension. 46, 1(2005), pp. 244-248. https://doi.org/10.1161/01.HYP.0000166723.07809.7e

[23] Van Bortel, L. M. et al. Non-invasive assessment of local arterial pulse pressure: comparison of applanation tonometry and echo-tracking. // Journal of Hypertension. $19,6(2001)$, pp. $1037-44$ https://doi.org/10.1097/00004872-200106000-00007

[24] Meinders, J. M.; Hoeks, A. P. Simultaneous assessment of diameter and pressure waveforms in the carotid artery. // Ultrasound Med Biol. 30, 2(2004), pp. 147-154 https://doi.org/10.1016/j.ultrasmedbio.2003.10.014

[25] Vermeersch, S. J. et al. Determining carotid artery pressure from scaled diameter waveforms: comparison and validation of calibration techniques in 2026 subjects. // Physiol Meas. 29, 11(2008), pp. 1267-1280. https://doi.org/10.1088/0967-3334/29/11/003

[26] Masson, I. et al. Characterization of arterial wall mechanical behavior and stresses from human clinical data. // Journal of Biomechanics. 41, (2008), pp. 2618-2627. https://doi.org/10.1016/j.jbiomech.2008.06.022

[27] Stålhand, J.; Klarbing, A.; Karlsson, M. Towards in vivo aorta material identification and stress estimation. // Biomechanics and Modeling in Mechanobiology. 2, (2004), pp. 169-186. https://doi.org/10.1007/s10237-003-0038-z

[28] Masson, I. et al. Carotid artery mechanical properties and stresses quantified using in vivo data from normotensive and hypertensive humans. // Biomech Model Mechanobiol. 10, 6(2011), pp. 867-882. https://doi.org/10.1007/s10237-010-0279-6

[29] Holzapfel, G. A.; Gasser, T. C.; Ogden, R. W. A new constitutive framework for arterial wall mechanics and a comperative study of material models. // Journal of Elasticity. 61, (2000), pp. 1-48. https://doi.org/10.1023/A:1010835316564

[30] Holzapfel, G. A.; Ogden, R. W. Modelling the layerspecific three-dimensional residual stresses in arteries, with an application to the human aorta. // J R Soc Interface. 7, 46(2010), pp. 787-799. https://doi.org/10.1098/rsif.2009.0357

\section{Authors' addresses}

Janez, Urevc

University of Ljubljana,

Faculty of Mechanical Engineering

Aškerčeva 6, 1000 Ljubljana, Slovenia

E-mail: janez.urevc@fs.uni-lj.si

Prof. Vojko Flis

University Clinical Center Maribor, Department of Vascular Surgery

Ljubljanska ulica 5, 2000 Maribor, Slovenia

E-mail: vojko.flis@ukc-mb.si

\section{Prof. Milan Brumen}

1) Faculty of Natural Sciences and Mathematics,

Faculty of Health Sciences, Faculty of Medicine

University of Maribor

Slomškov trg 15, 2000 Maribor, Slovenia

2) Institut Jožef Stefan

Jamova 39, 1000 Ljubljana, Slovenia

E-mail: milan.brumen@um.si

\section{Prof. Boris Štok}

University of Ljubljana,

Faculty of Mechanical Engineering

Aškerčeva 6, 1000 Ljubljana, Slovenia

E-mail: boris.stok@fs.uni-lj.si 